\title{
How to produce an absent-advantage in visual search
}

\author{
RONALD HÜBNER and PETER MALINOWSKI \\ University of Konstanz, Konstanz, Germany
}

\begin{abstract}
In a series of four experiments, we investigated the conditions under which target-absent responses are faster than target-present responses in visual search. Previous experiments have shown that such an absent-advantage occurs mainly for homogeneous distractors arranged in a regular pattern. From these results, it has been concluded that the absent-advantage is due to perceptual processes, such as grouping by similarity. Our data show that such processes are not sufficient. Rather, the absentadvantage is the result of interactions between perceptual and decisional processes. Certain perceptual conditions, such as randomizing stimulus patterns, lead to specific criteria settings that produce an absent-advantage. That such an account can explain our main results is demonstrated by modeling our data with a modified version of the Guided Search 2 model.
\end{abstract}

Visual search is one of the most important and influential experimental paradigms for investigating the basic properties of the visual system (see, e.g., A. Treisman \& Gelade, 1980; Wolfe, 1994). Usually, the participants' task is to search for a target item among a variable number of distractors, and the time needed to decide whether the target is present or not provides information as to how our visual system processes and represents the item features. A key issue in this paradigm is search functionsthat is, functions that describe how search time varies with the number of distractors. Usually, one obtains two such functions: one for target-present responses and one for target-absent responses.

When search functions are approximately linear, which is often the case, they can easily be characterized by their respective slope and intercept. Whereas the slopes are thought to reflect the efficiency with which item features are processed, intercepts are assumed merely to represent early visual processing and response processes that are fixed across conditions. Therefore, intercepts are considered to be relatively uninformative. Accordingly, in most visual search experiments, it was mainly the slopes that were of interest (e.g., Wolfe, 1998), whereas little attention was paid to the intercepts. Even the ratio of the intercepts has widely been ignored, unlike that of the slopes, which is regarded as highly informative. The present article differs from this view in that it concentrates on the intercepts for target-present and target-absent responses and their relations.

Usually, target-absent responses are slower than targetpresent ones. However, there are exceptions to this stan-

This research was supported by Research Grant Hu 432/6-1 from the Deutsche Forschungsgemeinschaft (DFG) to the first author. Correspondence concerning this article should be addressed to R. Hübner, Fachbereich Psychologie, Universität Konstanz, Fach D29, D-78457 Konstanz, Germany (e-mail: ronald.huebner@uni-konstanz.de). dard result. For instance, Humphreys, Quinlan, and Riddoch (1989) found that target-absent responses can be faster than target-present ones. Although such an absentadvantage represents a challenge to most visual search models, it has received little attention to date. This is surprising, because it could provide valuable information about visual search behavior. Therefore, the aim of the present paper is to investigate the conditions under which an absent-advantage occurs. However, before this issue is addressed experimentally, relevant results and concepts shall be reviewed briefly.

Many visual search experiments have examined the question of whether search proceeds in parallel across the visual field or in a serial manner. As the results have shown, when the target differs from the distractors by a unique feature, the slopes of the search functions are usually small. Such flat search functions were thought to indicate parallel search, which leads to a so-called pop-out of the target. On the other hand, when the target differs from the distractors by a specific conjunction of features, the search functions are usually steep, which has been assumed to indicate that search proceeded serially. This dichotomy of parallel and serial search is the core of the featureintegration theory (FIT; A. Treisman, 1988; A. Treisman $\&$ Gelade, 1980). With respect to serial search also absentresponses are important, since the relation between the slopes of the search functions for absent-responses and for present-responses can be used to test specific models. For instance, serial self-terminating search predicts a slope ratio of 2:1 (see, e.g., A. Treisman \& Gormican, 1988; A. Treisman \& Souther, 1985).

Meanwhile, the dichotomy between parallel and serial search has been questioned (e.g., Duncan \& Humphreys, 1989). There is increasing evidence that search efficiency (slopes) varies along a continuum and depends on various factors. A model that predicts such a continuum is Guided Search 2 (GS2; Wolfe, 1994). In this model, possible tar- 
get candidates are checked according to their activation strength until the target is found (respond present) or a given threshold is reached (respond absent). Although the main focus of GS2 is on the slopes for target-present functions, recently, Chun and Wolfe (1996) considered absent-responses in detail. However, in that article as well, the slopes, rather than the intercepts, were the focus of interest.

As has been mentioned, under certain circumstances, target-absent responses are faster than target-present ones (see, e.g., Humphreys et al., 1989). Such data can be predicted neither by FIT nor by GS2. To see under what conditions an absent-advantage might occur, let us consider Humphreys et al.'s procedure in more detail. In several experiments, they had participants search for an inverted $T$ among homogeneous upright $T \mathrm{~s}$, which were either arranged regularly on the circumference of an imaginary circle or placed irregularly in the visual field. It turned out that for regular patterns, absent-responses were faster than present-responses, whereas the opposite held for irregular patterns. Moreover, whereas the search times for present-responses increased linearly with set size, those for absent-responses remained constant.

However, regularity turned out not to be sufficient for obtaining an absent-advantage. In one experiment, in which the distractors consisted of upright, left-pointing, and right-pointing $T$ s, present-responses were always faster. Thus, for there to be an absent-advantage it seemed also to be essential that the distractors be homogeneous. To explain their data, Humphreys et al. (1989) assumed that similarity grouping between the items played a major role. Specifically, they relied on the theory proposed by Duncan and Humphreys $(1989,1992)$, which assumed that search performance depends on the similarity between the target and the distractors and on that between the distractors. On the basis of this theory, Humphreys and Müller (1993) developed a computational model (search via recursive rejection [SERR]) for explaining the absent-advantage. They assumed that similarity affects the amount of grouping between neighboring items and that groups of items are rejected as a whole until the target is found. Unfortunately, so far, the model predicts an absent-advantage even when there are two different types of distractors, which is at odds with the available data. To take homogeneity into account, and because the model produced too many misses, Humphreys and Müller introduced additional checking runs when no target was found. Since the probability of reruns was assumed to depend on the miss rate, which was larger for heterogeneous distractors, the absent-advantage vanished in this case.

Although the effect of distractor heterogeneity has been taken into account in the final version of the SERR model, its basic assumptions can be questioned. For instance, in a subsequent paper, Donnelly, Humphreys, and Riddoch (1991) were able to show that grouping by similarity, as implemented in the SERR model, is not necessary for an absent-advantage. They found an absent-advantage also for heterogeneous distractors as long as they could be grouped into a perceptual object. Donnelly et al. assumed that, in this case, the distractors were rejected as a whole.

It bears mention that there are other visual search models that also emphasize similarity grouping. For instance, Grossberg, Mingolla, and Ross's (1994) spatial and objects search (SOS) model follows the rationale that search is parallel within grouped regions but serial between those regions (see Pashler, 1987). However, although Grossberg et al. cite some results of Humphreys et al. (1989), the SOS model cannot account for faster absent-responses.

This brief overview shows that an absent-advantage is still a challenge for current visual search models. Even the SERR model, which was developed especially for such cases, explains the empirical results only partly. On the other hand, if one considers the currently available data, it is not quite clear under what circumstances an absent-advantage occurs. Although homogeneity and regularity seem to be important, they are neither necessary nor sufficient. For instance, Wang, Cavanagh, and Green (1994) used regular homogeneous patterns but did not observe an absent-advantage. They employed the letters $S, N$, and $Z$, instead of $T$. However, it is unlikely that this difference is responsible for the absence of the absentadvantage. Thus, the question is still open as to what exactly is essential for producing an absent-advantage.

A hint for a possibly important factor can be obtained by considering Humphreys et al.'s (1989) Experiment 3, in which no absent-advantage was observed, even though the patterns were homogeneous and regular. Its main difference from experiments in which an absent-advantage did occur was that the trials with regular patterns were not mixed with those with irregular ones. This suggests that mixing regular and irregular patterns within a block of trials could be crucial for obtaining an absent-advantage. If this hypothesis holds, it could be concluded that not only perceptual processes, such as grouping, but also decisional mechanisms are involved in the absent-advantage. Thus, a series of experiments was conducted to investigate this issue. Whereas our first experiment served to replicate the absent-advantage, in the subsequent experiments decisional mechanisms were examined.

\section{EXPERIMENT 1}

The first experiment served to replicate the absentadvantage as obtained by Humphreys et al. (1989) and, thereby, to validate our method. We tried to use the same procedure as Humphreys et al., except that, instead of a fixed number of irregular patterns being used, the items were randomly positioned for constructing an irregular pattern on the corresponding trials.

\section{Method}

Participants. Eight persons (6 male and 2 female), who ranged in age from 23 to 33 years (mean, 26.8), participated in the experiment. All reported normal or corrected-to-normal vision.

Apparatus. The stimuli were presented on a 19-in. color monitor with a resolution of $1,280 \times 1,024$ pixels. A personal computer $(\mathrm{PC})$ served for controlling stimuli presentation and response registration. 


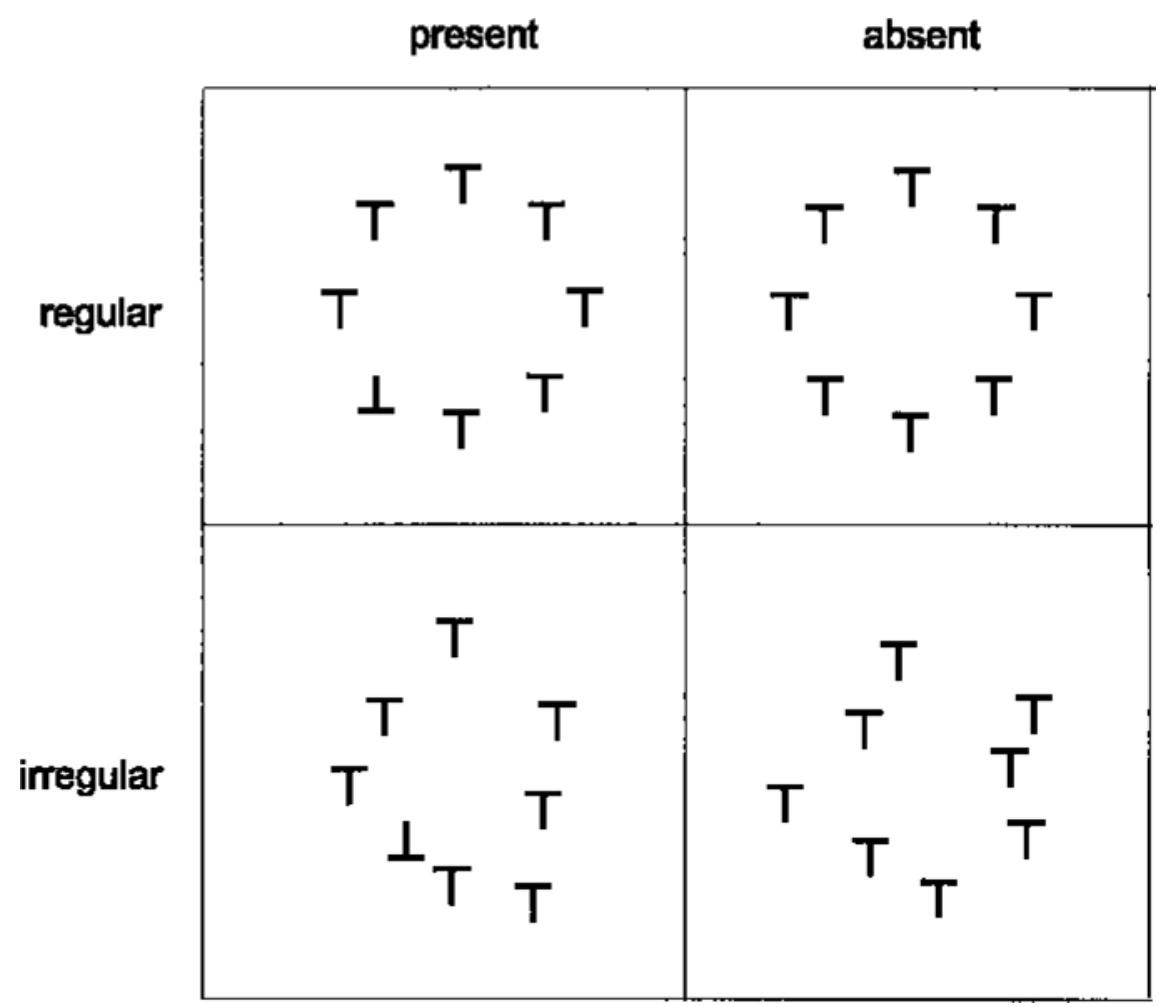

Figure 1. Examples of regular and irregular patterns used in Experiment 1.

Stimuli. Elements were upright and inverted $T$ s subtending $0.7^{\circ}$ $\times 0.7^{\circ}$ of visual angle at a viewing distance of $127 \mathrm{~cm}$, which was held constant with a head-and-chin rest. The stimuli were white $\left(\sim 40 \mathrm{~cd} / \mathrm{m}^{2}\right)$ and appeared on a black background $\left(\sim 0.3 \mathrm{~cd} / \mathrm{m}^{2}\right)$. Targets and distractors were inverted and upright $T \mathrm{~s}$, respectively.

Three set sizes of four, six, and eight elements were used. The elements were arranged on the circumference of an imaginary circle with a radius of $2.2^{\circ}$. In the regular patterns the elements were equally spaced, whereas in the irregular condition each element was placed randomly around the position it would have had in a regular pattern. The maximum amount of displacement for each element was $0.4^{\circ}$ in the horizontal and vertical directions, respectively. See Figure 1 for an example.

Procedure. Each trial started with a fixation cross that was presented centrally on the screen for $400 \mathrm{msec}$. Immediately afterward, the stimulus pattern appeared and remained present until a response was given. After the response, a blank interval of 1,000 msec was inserted before the next trial began. Errors were signaled by a tone. The participants responded with the index and middle fingers of their right hand for target-present and target-absent judgments, respectively, where the response-to-f inger mapping was balanced across subjects. Altogether, there were 12 different conditions (targetpresent/target-absent $\times$ regular/irregular pattern $\times 3$ set sizes) with 72 trials each. They were run in nine blocks of 96 trials, with 4 warm-up trials at the beginning of each block. Each condition occurred eight times in each block in random order.

\section{Results}

The mean latencies of correct responses were subjected to a within-subjects analysis of variance (ANOVA), with target presence (present or absent), regularity (regular or irregular), and set size (four, six, or eight) as factors.
The analysis revealed a significant regularity effect $[F(1,7)=18.7, p<.01]$. Regular patterns produced shorter latencies than irregular ones (572 msec vs. $594 \mathrm{msec}$ ). However, there was a significant interaction between regularity and presence $[F(1,7)=8.05, p=.025]$. The absent-advantage was larger for regular patterns (557 vs. $587 \mathrm{msec}$ ) than for irregular ones (587 vs. $601 \mathrm{msec})$.

Also, the set-size factor was significant $[F(2,14)=6.26$, $p<.05]$. The latencies increased with set size $(570,584$, and $596 \mathrm{msec})$. However, set size interacted with regularity $[F(2,14)=4.79, p=.026]$. The latency increase with set size was larger in the irregular condition than in the regular one (regular: 562, 577, and $587 \mathrm{msec}$; irregular: 578,591 , and $614 \mathrm{msec}$ ).

Finally, there was a reliable interaction between set size and presence $[F(2,14)=5.87, p<.05]$. The absentadvantage was larger with set size $4(38 \mathrm{msec})$ than with the other two set sizes (10 and $17 \mathrm{msec}$ ). The data are displayed in Figure 2.

Errors occurred, on average, in $2.3 \%$ of the trials. The rates can also be seen in Figure 2. Since they were quite small and since there seem to be no speed-accuracy tradeoff effects, they were not further analyzed.

\section{Discussion}

Our results replicate those of Humphreys et al. (1989). There was a large absent-advantage for regular patterns. However, even for irregular patterns, the absent-responses 


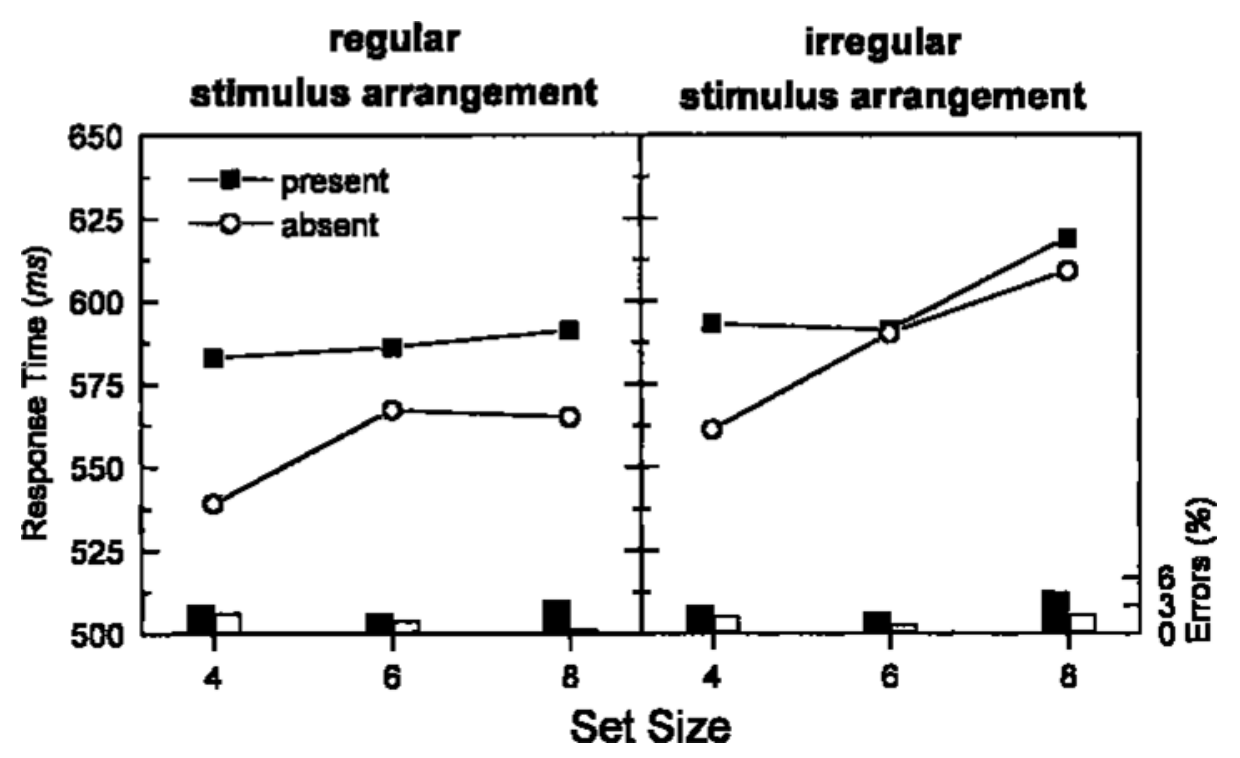

Figure 2. Results of Experiment 1.

were slightly faster than the present-responses, which is probably due to our restricted distortion of regularity. Surprisingly, the absent-advantage was largest for the smallest set size. This result is difficult to explain with similarity grouping. Grouping should be stronger when more elements are present. However, one might argue that with four elements a "square" can be perceived as a good Gestalt (see Donnelly et al., 1991). In any case, our method proves to be appropriate for examining conditions that affect the absent-advantage.

\section{EXPERIMENT 2}

In the following experiment, we tested the hypothesis that an appreciable absent-advantage for homogeneous and regular patterns occurs only when corresponding trials are mixed with those on which irregular patterns are presented. As was pointed out in the introduction, this seems to be a plausible explanation for the missing absentadvantage in Experiment 3 of Humphreys et al. (1989). Although the patterns were homogeneous and regular in that experiment, they were not mixed with irregular patterns. On the other hand, the patterns were mixed in all the experiments in which an absent-advantage occurred. To test our hypothesis, regularity was blocked in one condition and randomized in another one.

\section{Method}

Twelve participants ( 5 male and 7 female), who ranged in age from 19 to 35 years (average, 25.8 years), were involved in the experiment. All reported normal or corrected-to-normal vision.

The equipment and stimuli were the same as those in Experiment 1 . Four factors were varied: target presence (present or absent), set size (four, six, or eight), regularity (regular or irregular), and context (constant regularity or mixed regularity). The first three factors were randomized in each experimental block. Concerning the last factor, half of the participants started with the mixed- regularity blocks and then received the constant-regularity blocks. For the other half of the participants, this order was reversed. As in the previous experiment, set size was randomized.

Altogether, there were 24 different conditions, which were run in two 1-h sessions. The trials with mixed regularity were distributed over nine blocks of 96 trials. Altogether, there were 72 trials per condition. Trials with constant regularity were split into eight blocks of 96 trials, resulting in 64 trials per condition. After a practice block, half of the participants started with regular patterns (four blocks), whereas the other half started with irregular ones (four blocks). In all other respects, the procedure was the same as that in Experiment 1.

\section{Results}

Response Times. The mean latencies for correct responses were entered into a within-subjects ANOVA, with presence (present or absent), regularity (regular or irregular), context (constant regularity or mixed regularity), and set size (four, six, or eight) as factors. The mean response times are displayed in Figure 3.

Of the main effects, only set size was significant $[F(2,22)=35.93, p<.001]$. However, there was a reliable interaction between set size and regularity $[F(2,22)=$ $4.18, p<.05]$. Response times increased more with increasing set size for irregular patterns than with that for regular ones (irregular: 493, 502, and $515 \mathrm{msec}$; regular: 495, 498, and $505 \mathrm{msec}$ ). Furthermore, there was a significant two-way interaction between regularity and presence $[F(1,11)=5.95, p<.05]$. However, the three-way interaction between regularity, presence, and context also was significant $[F(1,11)=8.19, p<.05]$. As can be seen by inspecting Figure 3, an appreciable absent-advantage occurred for regular patterns only when regularity was mixed. Finally, there was a reliable three-way interaction between set size, presence, and regularity $[F(2,22)=3.99$, $p<.05]$. Whereas the set-size effect for present-responses was hardly affected by regularity, the search functions 


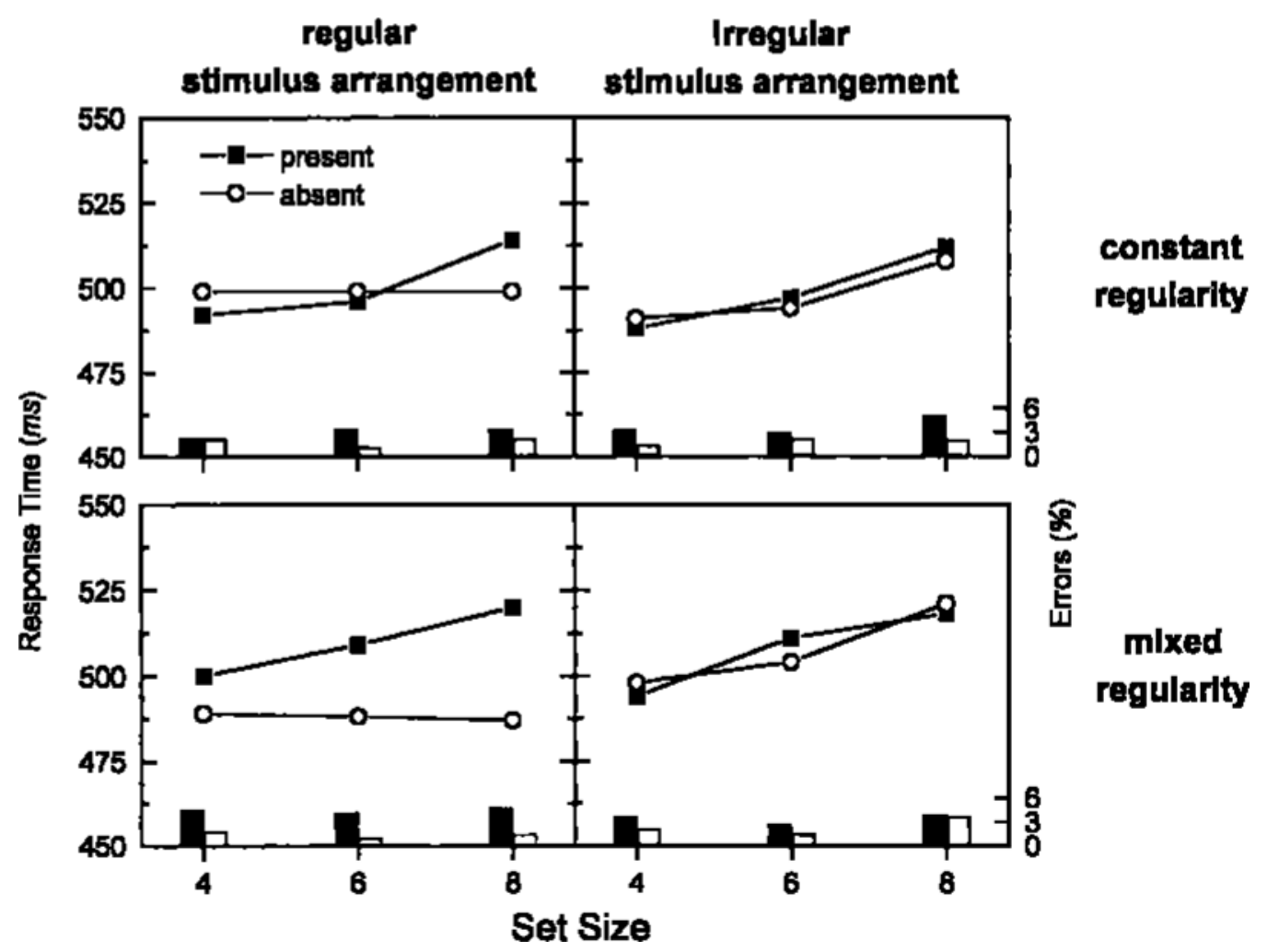

Figure 3. Mean response times for the different conditions in Experiment 2.

for absent-responses increased in slope with irregular patterns.

Errors. Errors occurred in $2.76 \%$ of the trials. The mean error rates were subjected to an ANOVA analogous to that for the latencies. There was a main effect of set size $[F(2,22)=5.15, p<.05]$, with error rates of $2.65 \%$, $2.36 \%$, and $3.29 \%$ for set sizes of four, six, and eight, respectively. Also, the presence factor produced a significant main effect $[F(1,11)=25.8, p<.001]$. There were fewer false alarms $(1.89 \%)$ than misses $(3.62 \%)$. Furthermore, there was a significant three-way interaction of regularity, presence, and experimental context $[F(1,11)=$ $11.8, p<.01]$. If one considers responses to regular patterns, the miss rate increased $(3.00 \%$ vs. $4.40 \%)$ and the false alarm rate decreased (1.87\% vs. $1.36 \%$ ) under mixed regularity, as compared with performance under constant regularity. On the other hand, the effect was reversed for irregular patterns (misses, $3.73 \%$ vs. $3.36 \%$; false alarms, $1.91 \%$ vs. $2.45 \%$ ). The error rates are shown in Figure 3.

\section{Discussion}

The results show that absent-responses to one type of pattern can be strongly affected by the occurrence of other types in the experimental block. Similar to Experiment 1 , there was a large absent-advantage when regular and irregular patterns were mixed within a block of trials. However, no appreciable absent-advantage occurred when regularity was constant.
By inspecting the data, it can be seen that regular patterns produced flat search functions for absent-responses. This holds for constant as well as for mixed regularity and is different from the previous experiment. In any case, the intercept of the search functions varied with context. With respect to regular patterns, mixing both pattern types decreased the intercept for absent-responses and increased that for present-responses. These shifts of the search function led to an absent-advantage. Thus, our data demonstrate that the absent-advantage is not due solely to perceptual factors, such as grouping. Rather, decisional factors also seem to be important. The strategy effect is also reflected in the error data. They indicate that speeding up the absent-decisions for the regular pattern in the mixed condition was done at the expense of an increased number of misses.

How can the shifts of the search functions be explained? By means of a formal model, we will show that they can be accounted for by assuming different criteria in the mixed condition.

\section{A FORMAL ACCOUNT OF THE ABSENT-ADVANTAGE}

As was mentioned in the introduction, the current search models, except SERR, cannot explain an absentadvantage. Since our data suggest that whole-group rejection is less important than is assumed by the SERR 
model, we will show how GS2 (Wolfe, 1994), currently one of the most prominent and detailed visual search models, can be modified to allow faster absent-responses than present-responses. In GS2, the items produce activations on an internal activation map. The activation caused by an item does not depend only on its features, but also on the feature differences and on the spatial distance to its neighboring items. The core assumption of GS2, however, is that the items are checked according to their activation strength. That is, attention is guided first to the item with the highest activation. If this item is the target, a present-response is triggered. Otherwise, attention moves to the item with the next highest activation. This loop proceeds until the target is found or the activation of the current item is below an activation threshold. In the latter case, a target-absent response is triggered. With respect to our objective, it is important to note that, since the item with the highest activation is checked in any case, GS2 cannot predict that absent-responses will be faster than present-responses.

How can GS2 be modified to produce an absentadvantage? A simple way would be to assume that guided search only starts when the activation threshold is passed within a certain limit of time. As a consequence, if a pattern produces only low activations that do not reach the threshold before a prespecified deadline, an absentresponse can be triggered relatively early. A similar mechanism is already part of GS2. Since the target-checking process does not produce errors in GS2, a mechanism was required that accounts for false alarms. Therefore, it is assumed that on a few trials, when no activation crosses the activation threshold or when no target is found within a certain period of time, search is terminated by an "educated guess" (see Chun \& Wolfe, 1996; Wolfe, 1994), where a certain percentage of these guesses are absentresponses. Chun and Wolfe assume that guesses are due to "boredom, fatigue, frustration, anticipation, etc." (p. 50). Here, we propose that such early decision mechanisms are an important part of the visual search process.

It can be an efficient strategy to respond absent when no activation passes the threshold before a deadline. When activations pass the threshold relatively early, this could be due to the target or the distractors. Thus, checking is necessary in any case. However, when all activations remain below the threshold before the deadline is reached, it is likely that there is no target. Thus, we argue that such a deadline is responsible for the absent-advantage. To demonstrate that such an account is possible, we modified a formalized version of the GS2 model (Hübner, in press) and calculated corresponding search times and errors. Details of the formal model are described in the Appendix.

For our calculations, a number of parameters had to be specified. First of all, we assumed that target and distractor activations are normally distributed random variables. In a first step, we fitted the parameters by visual inspection to our data for blocked regular patterns. This resulted in an offset of $470 \mathrm{msec}$ and a search time of $50 \mathrm{msec}$ per item. As the mean and standard deviation of the target activation we chose 445 and 35 , respectively.
The corresponding parameters for the distractor activation were 300 and 60 . That the variance of the target activation was smaller than that of the distractor activations is in line with the simulations of Chun and Wolfe (1996). To produce search functions that were similar to our empirical functions (in Experiment 2), we had also to take into account that they were relatively flat for regular patterns, especially for absent-responses. Therefore, we assumed that this was due to a decreasing variance of the distractor activations with increasing set size. This is a reasonable assumption, because the distance between the items decreased with increasing set size. Thus, in our calculations, the standard deviation was divided by $(0.80+$ setsize $/ 20)$. Furthermore, it was assumed that the mean of the target distribution decreases slightly with set size. This was necessary to avoid search functions with negative slopes for present-responses. The decreasing target activation might be motivated by the fact that the target is increasingly integrated in the circular form with decreasing distance between the items (see Prinzmetal \& Banks, 1977). This is a point at which grouping might play a role. For instance, it could be possible that processes at a higher scale affect the activations. However, it is also conceivable that specific interactions between early feature detectors reduce the target activation. In any case, in our calculations, the mean of the target activation was divided by $(0.98+$ setsize/200).

So far, our assumptions are compatible with GS2 and are concerned with merely perceptual effects that affect the slopes of the search functions. To obtain an absent-advantage, we did not use a deadline, as was suggested above. Rather, for simplicity, we introduced a nothreshold and assumed that, when all activations are below that threshold, an absent-response is triggered immediately without any search. It is clear that a sufficient number of such fast absent-responses in a block of trials can account for an absent-advantage.

In a similar manner, we produced false alarms. Instead of educated guesses, a yes-threshold was introduced. When an activation passes this threshold, a presentresponse occurs immediately. Thus, guided search takes place only when activations cross the no-threshold but not the yes-threshold.

Although preliminary threshold values were assumed for choosing the general parameters of the model, in the next step they were optimized by fixing the general parameters and fitting the model to our search times and errors by means of a minimization algorithm (the function "fminsearch" from MATLAB). The program found a nothreshold of 370, an activation threshold of 389, and a yes-threshold of 454 . The resulting theoretical data produced by the model with these parameters can be seen in the upper panel of Figure 4.

To obtain an absent-advantage, we fixed the general parameters and the activation threshold and fitted the other two thresholds to the data from the regular patterns in the mixed condition. Since we did not allow the no-threshold to be larger than the activation threshold, we obtained a no-threshold of 389 and a yes-threshold of 469 . The cor- 


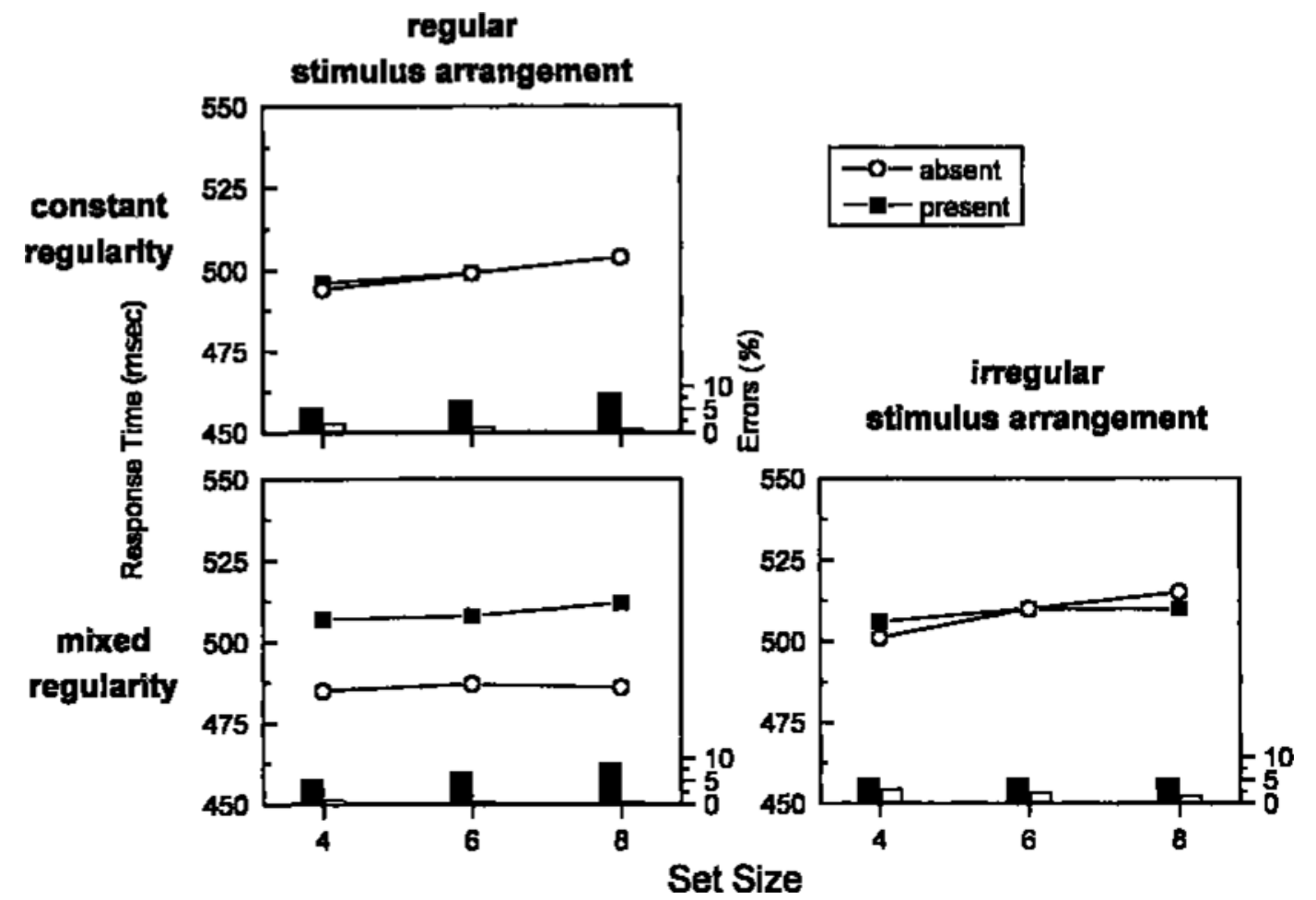

Figure 4. Theoretical data obtained with a modified version of the Guided Search 2 model. The parameters were fitted to the data of Experiment 2.

responding data can be seen in the lower left panel of Figure 4 . As can be seen, by shifting these two thresholds an absent-advantage occurs.

Finally, we wanted to fit the model to the data for the irregular patterns in the mixed condition. We assumed the same thresholds as those for the regular patterns and fitted merely perceptual parameters. Specifically, the mean and the standard deviation of the distractor activations were used as free parameters, where the mean did not vary with set size in this case. With our minimization program, we obtained values of 62 and 320, respectively. The corresponding results can be seen in the lower right panel of Figure 4.

The calculations demonstrate that decisional processes seem to play an important role for the absent-advantage. Under certain conditions, thresholds can be adjusted in such a way that an absent-advantage results for a specific pattern type. One of these conditions is the mixing of regular and irregular patterns.

Why would the mixing of pattern types have led to such specific adjustments of the involved thresholds? Our computations suggest that the irregular arrangement of the items produced a different distribution of distractor activations, relative to a regular arrangement. This is presumably due to the variable spatial distances between the items, which modulated the activations in a certain way. In any case, it is conceivable that, when only one distractor distribution is effective in a block of trials, the participants adjust their thresholds accordingly. On the other hand, when two distributions are mixed across trials, the thresholds are adjusted to cope with both distributions simultaneously.

If our account is correct, it should be possible to produce an absent-advantage by a method other than arranging the items irregularly. The crucial condition would be to use a pattern that produces a specific distribution of the distractor activations. Such an approach was applied in the next experiment.

\section{EXPERIMENT 3}

In this experiment, we again used homogeneous regular patterns. However, instead of mixing these patterns with irregular ones, they were mixed with heterogeneous patterns. The latter patterns were constructed by replacing some distractors with items that were more similar to the target. Specifically, we used a double cross as the target and squares as one type of distractors. The other distractor type were double crosses that differed from the target merely by the amount of indent. Examples of the different patterns can be seen in Figure 5.

According to our hypothesis, mixing the homogeneous patterns with the heterogeneous ones should produce an absent-advantage. We assumed that the heterogeneous distractors correspond to an activation distribution with an increased variance, relative to the distribution of homogeneous distractors, and with a mean closer to that of the target. Thus, in constant conditions, the participants 


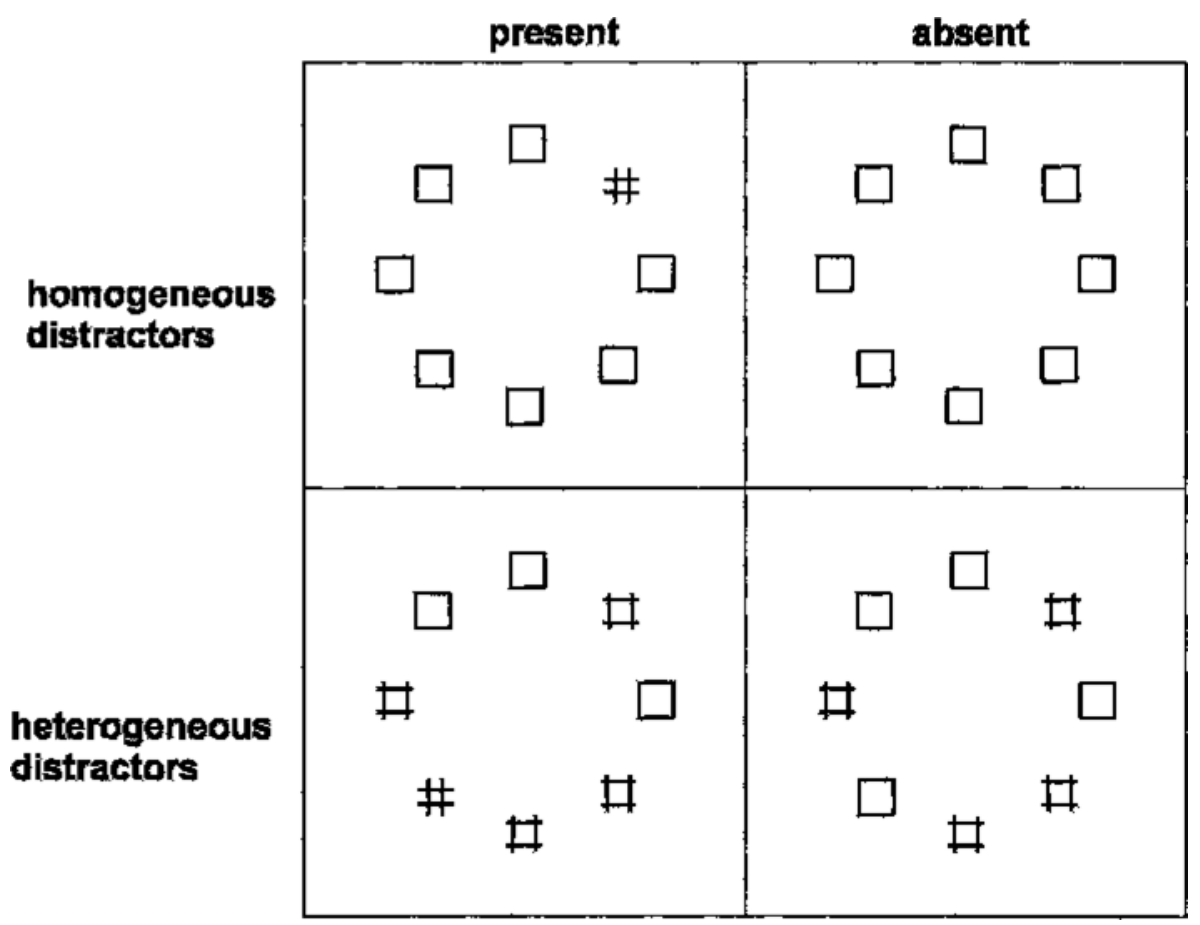

Figure 5. Examples of the stimuli with a set size of eight, used in Experiment 3.

should use different thresholds for heterogeneous patterns than for homogeneous ones. We expected that they would use an increased no-threshold and yes-threshold for the heterogeneous patterns in the constant condition and also when both patterns were mixed across trials. This should produce an absent-advantage for the homogeneous patterns in the mixed condition.

\section{Method}

Eight persons ( 6 female and 2 male), who ranged in age from 20 to 35 years (mean, 24.5), participated in the experiment. All reported normal or corrected-to-normal vision.

The stimuli were presented on a 20-in. color-monitor (Sony) with a resolution of $1024 \times 768$ pixels. A PC served for controlling stimuli presentation and response registration.

Items were squares and double-crosses subtending $0.7^{\circ} \times 0.7^{\circ}$ of visual angle at a viewing distance of $105 \mathrm{~cm}$, which was held constant with a head-and-chin rest. They were white and appeared on a black background. The target and distractors were constructed from four lines of identical length. The first distractor type was a square, whereas the second type was a wide double cross, which was constructed by slightly indenting (i.e., $8.3 \%$ of the line length on each side) the four lines of the square. The target was always a narrow double cross. It differed from the second distractor type by the amount of indention ( $16.7 \%$ of the line length on each side). See Figure 5 for examples.

Set sizes of four, six, and eight items were used. The items were arranged on the circumference of an imaginary circle with a radius of $2.2^{\circ}$. On each trial, the distractors were randomly assigned to the possible equally spaced positions on the imaginary circle. In the homogeneous patterns, all the distractors were squares, whereas in the heterogeneous condition, squares and wide double crosses served as distractors. Heterogeneous patterns with no target had an equal number of each distractor type. On target-present trials, a randomly chosen distractor was replaced by the target.
Four factors were varied: target presence (present or absent), set size (four, six, or eight), homogeneity (homogeneous or heterogeneous), and context (constant homogeneity or mixed homogeneity). The first two factors were randomized in each experimental block. Depending on the context factor, homogeneity was either blocked or mixed. Half of the participants started with the mixedhomogeneity blocks in the first session and then continued with constant-regularity blocks in the second session. For the other half of the participants, this order was reversed.

There were 24 different conditions, which were run in two 1-h sessions. The trials for the mixed-homogeneity condition were distributed over nine blocks of 96 trials. Altogether, there were 72 trials per condition. Trials for the constant-homogeneity condition were split into eight blocks of 96 trials, resulting in 64 trials per condition. In all other respects, the procedure was the same as that in Experiment 2.

\section{Results}

Response times. The mean latencies of correct responses were entered into a within-subjects ANOVA, with presence (present or absent), homogeneity (homogeneous or heterogeneous), context (constant homogeneity or mixed homogeneity), and set size (four, six, or eight) as factors. The mean response times are displayed in Figure 6.

The data analysis revealed a significant effect of set size $[F(2,14)=7.91, p<.01]$. Also, the homogeneity factor produced a significant effect $[F(1,7)=20.9, p<.01]$. However, there was a significant interaction between these factors $[F(2,14)=4.99, p<.05]$. For homogeneous patterns, the response times were independent of set size (443, 442, and 444 msec for set size 4,6 , and 8, respectively), whereas they increased with set size for heterogeneous patterns $(493,495$, and $509 \mathrm{msec})$. 


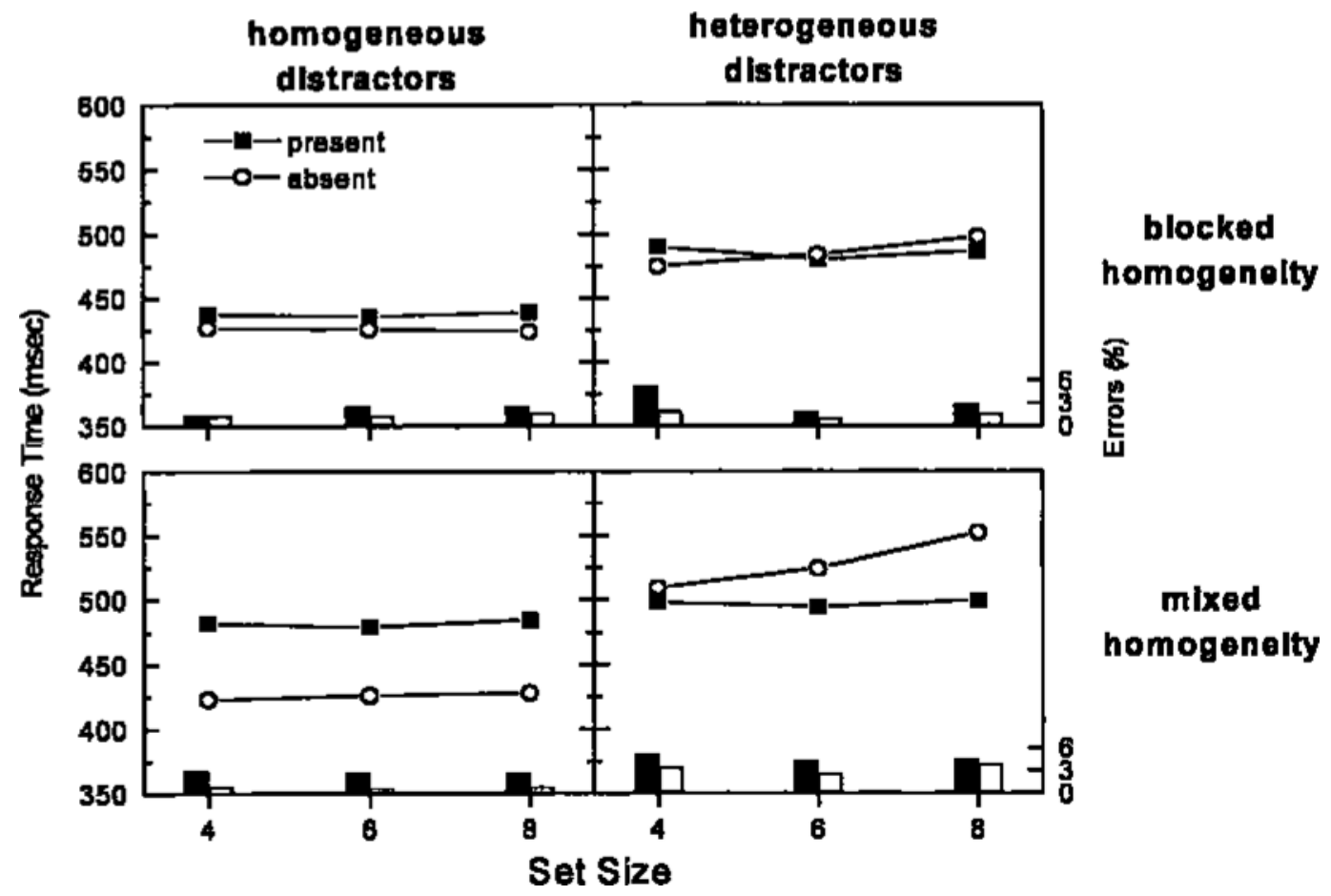

Figure 6. Mean response times and error rates for the different conditions in Experiment 3.

Furthermore, there was a reliable interaction between homogeneity and presence $[F(1,7)=85.0, p<.001]$. However, there was also a three-way interaction of homogeneity, presence, and context $[F(1,7)=9.70, p<$ $.05]$. Figure 6 shows that an appreciable absent-advantage (about $56 \mathrm{msec}$ ) occurred only for homogeneous distractors in the mixed condition.

Errors. Errors occurred in $2.48 \%$ of the trials. The mean error rates are shown in Figure 6. These data were subjected to an ANOVA analogous to that for the latencies. There was a main effect of presence $[F(1,7)=10.38$, $p<.05]$, indicating that more errors occurred on presenttrials than on absent-trials ( $3.19 \%$ vs. $1.78 \%)$. Also, homogeneity produced a significant main effect $[F(1,7)=$ $17.02, p<.005]$. However, there was a significant interaction between context and homogeneity $[F(1,7)=8.03$, $p<.05]$. Whereas the error rates for homogeneous patterns were independent of context $(1.82 \%$ vs. $1.77 \%$, for constant and mixed homogeneity, respectively), those for heterogeneous patterns increased in the mixed condition $(2.41 \%$ vs. $3.94 \%)$.

\section{Discussion}

As was expected, in the mixed conditions, there was a large absent-advantage for homogeneous patterns. However, this effect was dependent on the mixing of the two pattern types. In the constant condition, the absentadvantage was negligible. This shows again that it is important to take decisional processes into account. If only data with mixed pattern types had been collected, one would presumably have claimed that homogeneity as such produces a strong absent-advantage. Moreover, this effect would probably have been attributed to grouping. Fortunately, we also considered constant conditions and, therefore, know that these interpretations are not valid.

By using the same procedure as that for the data from the previous experiment, we fitted our model to the present data also. As general parameters, we chose an offset of $420 \mathrm{msec}$ and a search time per item of $50 \mathrm{msec}$. For the homogeneous patterns in the constant conditions, we obtained values for the target mean and variance of 460 and 35 , respectively. The corresponding values for the distractors were 280 and 60 . With the minimization algorithm, we found a value of 392 for the no-threshold and 394 for the activation threshold. For the yes-threshold, we obtained a value of 448 .

In the next step, we fitted the model to the data for the homogeneous patterns in the mixed condition. Since the response time for the absent-responses hardly changed between the two conditions, all the parameters were fixed, except the yes-threshold. Increasing this threshold increases the response time on target-present trials. Since the program increased the threshold without converging to a minimum, we set the value to 540 .

For the heterogeneous patterns in the mixed condition, we fixed all the parameters, except the mean and standard deviation of the distractor distribution. For simplicity, we did not use an individual distribution for each type of distractor items within the pattern but, rather, a common distribution. The program found values of 86 and 346 for the mean and standard deviation, respectively. Theoretical data for these different parameter sets can be seen in 


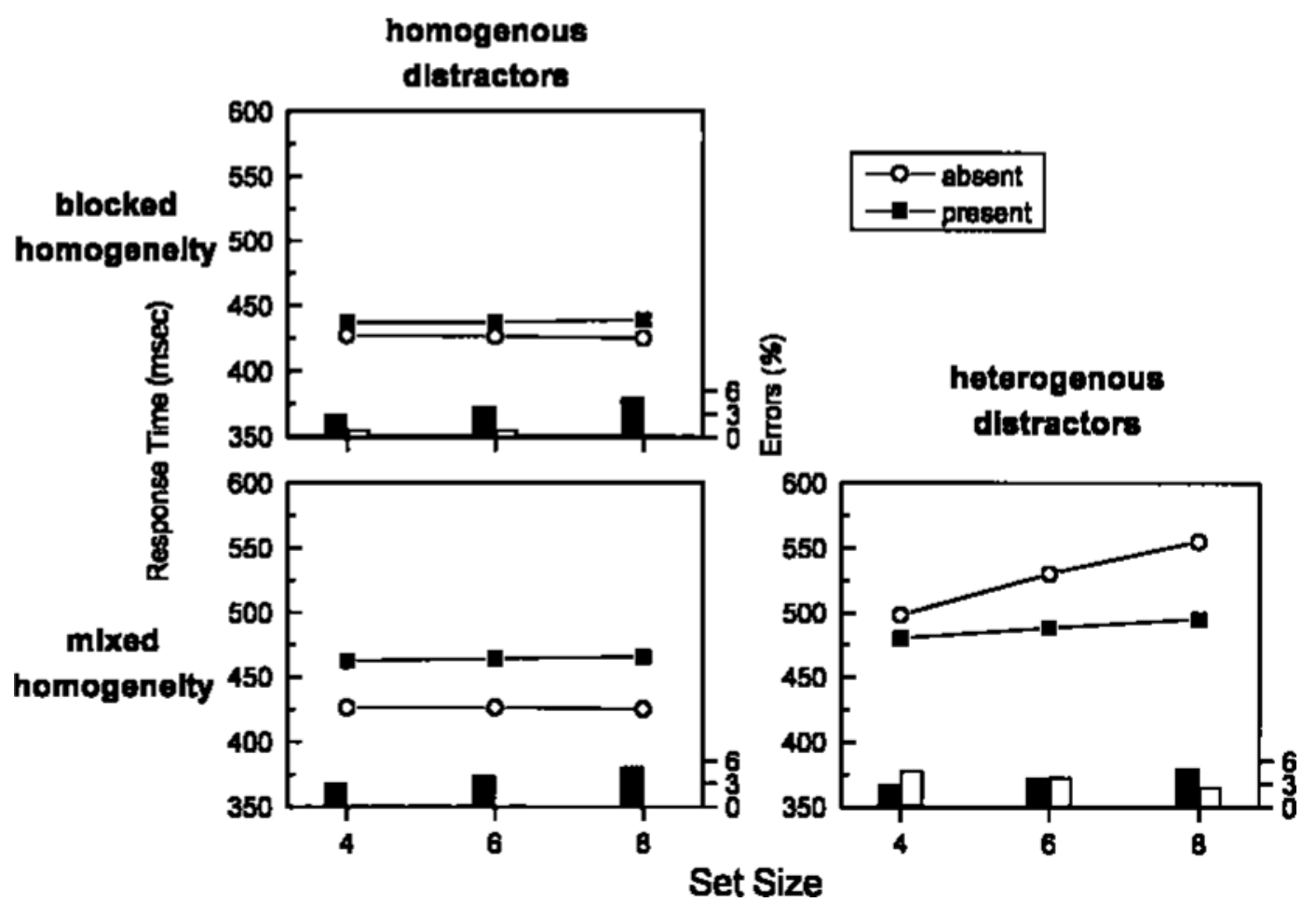

Figure 7. Theoretical data obtained by fitting the model to the data of Experiment 3.

Figure 7 . The data and our model fitting suggest that, in the mixed condition, the participants used the same yesthreshold as that for the heterogeneous patterns in the constant condition but that they used the no-threshold from the constant condition with homogeneous patterns.

\section{EXPERIMENT 4}

The patterns in the previous experiment were always regular. Is this a necessary condition for a strong absentadvantage with homogenous distractors and mixed patterns? When the distributions of distractor activations and their mixing across trials is important for the adjustment of a no-threshold or deadline, the absent-advantage should not depend on regularity, although regular patterns might be helpful under some circumstances. Since we used specific items for producing an absent-advantage in the last experiment, the effect of regularity should have been negligible. Therefore, we should obtain an absentadvantage with these items even for irregular patterns. This prediction was tested in this experiment.

\section{Method}

Four persons ( 1 female and 3 male), who ranged in age from 20 to 44 years, participated in the experiment. All reported normal or corrected-to-normal vision.

The apparatus, the procedure, and the stimuli were the same as those in the previous experiment, except that the patterns were irregular and that homogeneity was always randomized. Irregularity was obtained by displacing the elements, where the maximum amount of displacement for each element was $0.8^{\circ}$ in the horizontal and vertical directions. Overlaps of the items were prevented.

\section{Results and Discussion}

The mean latencies of correct responses were subjected to a within-subjects ANOVA, with presence (present or absent), homogeneity (homogeneous or heterogeneous), and set size (four, six, or eight) as factors. The data analysis revealed a significant effect of set size $[F(2,6)=$ $6.54, p<.05]$. Also, the homogeneity factor produced a significant effect $[F(1,3)=19.5, p<.05]$. However, and most important, there was a significant interaction between homogeneity and presence $[F(1,3)=25.6, p<$ $.05]$. As can be seen in Figure 8, although the presentresponses were faster for the heterogeneous patterns, there was a large absent-advantage for the homogeneous patterns. Errors are also shown in Figure 8. They were not further analyzed.

Thus, as was expected, the results show that the absentadvantage can be produced just by mixing homogeneous patterns with heterogeneous ones, irrespective of regularity.

\section{GENERAL DISCUSSION}

The aim of the present experiments was to examine conditions that produce an absent-advantage in visual search. Such an effect had originally been observed for homogeneous distractors arranged on the circumference 


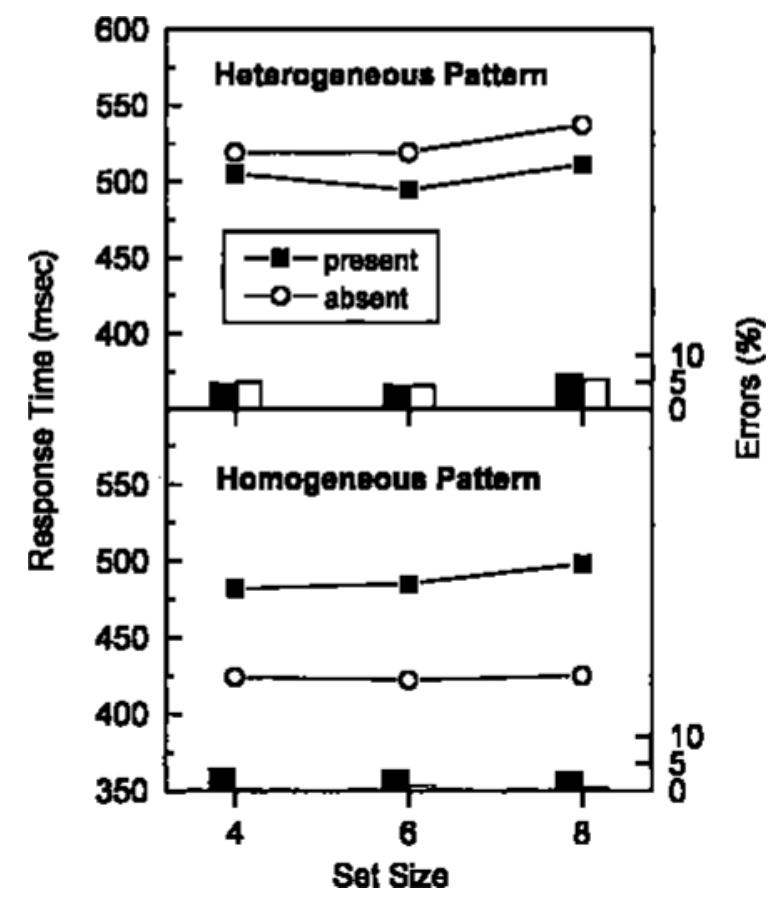

Figure 8. Results from Experiment 4. All the patterns were irregular, and homogeneity was always randomized.

of an imaginary circle (e.g., Humphreys et al., 1989). Since no absent-advantage had been obtained with irregular patterns and/or heterogeneous distractors in these experiments, regularity and homogeneity had been considered to be the crucial factors for an absent-advantage. Furthermore, it had been concluded that these factors allow whole-pattern rejection by means of similarity grouping (e.g., Humphreys \& Müller, 1993; Humphreys et al., 1989; Müller, Humphreys, \& Donnelly, 1994). However, there were data that did not support this hypothesis (e.g., Donnelly et al., 1991; Wang et al., 1994).

Thus, it was still unclear what factors are responsible for an absent-advantage. In any case, the data suggested that perceptual processes are not sufficient for producing absent-responses that were faster than present-responses. If one considers the conditions under which an absentadvantage has been observed for a certain pattern type, it is obvious that the patterns were always mixed with other pattern types across trials. This indicates that decision processes are involved in the absent-advantage. To examine their contribution in detail, the role of pattern mixing was investigated systematically in the present article. We compared the performance between conditions with constant pattern types and those with mixed pattern types. As was expected, when regular patterns with homogenous distractors were blocked, there was no absentadvantage. However, when regular and irregular patterns were mixed across trials, an absent-advantage was observed for the former type (Experiment 2). This shows again that homogeneous distractors arranged in a regular pattern are not sufficient for an absent-advantage. What is also necessary is that these patterns are mixed with, for instance, irregular patterns.

Our results cannot be accounted for by models, such as the SERR model, that explain the absent-advantage mainly by means of specific perceptual processes. Thus, an alternative account, based on the GS2 model, has been provided here. One of the basic features of GS2 is that the items are checked according to their activation strength on an activation map until the target is found or an activation threshold is reached. However, this mechanism cannot produce faster absent-responses than presentresponses. Therefore, we introduced a no-threshold and assumed that an absent-response is triggered immediately when no activation is above this threshold. This threshold allows fast absent-responses and, consequently, can lead to an absent-advantage. In addition, we introduced a yesthreshold and assumed that a present-response is triggered immediately when there is an activation that is higher than this threshold. Since this also occurs on some absenttrials, the yes-threshold is responsible for false alarms.

Thus, according to our account, guided search takes place only when some activations are above the nothreshold but no activation is higher than the yesthreshold. This approach is similar to ideas developed in the area of matching tasks, where it has to be explained that same judgments are often faster than different judgments (e.g., Bamber, 1969; Krueger, 1978). For instance, Krueger assumed that same-different judgments are based on the output of a difference counter. Very low and very high counts lead to immediate same responses and immediate different responses, respectively. When the count is intermediate, it does not provide sufficient evidence for an immediate response, and further processing is necessary.

Given the additional thresholds, the GS2 model can explain the absent-advantage. Our modeling suggests that homogeneous and regularly arranged distractor items produce low activations with small variance on the activation map, whereas the variance and, perhaps, also the mean is increased in the irregular condition. With blocked patterns, the thresholds are adjusted individually for each type, so no or, at least, no appreciable absent-advantage occurs. However, when the different pattern types are mixed, the participants adjust their thresholds to cope with these types simultaneously. Indeed, there is other evidence that criteria cannot be adjusted rapidly on a trialby-trial basis but remain largely constant within a block of trials (e.g., Strayer \& Kramer, 1994; M. Treisman \& Williams, 1984). In our case, the adjustments lead to an absent-advantage for certain pattern types. For instance, our modeling of the data from Experiment 2 suggests that the no-threshold was increased in the mixed condition, relative to the condition with constant regular patterns. This increased the number of fast absent-responses for the regular patterns and, therefore, produced an absentadvantage. On the other hand, the yes-threshold also was increased, which slowed the mean response time on targetpresent trials. This increased the absent-advantage. 
That our account holds more generally, and not only for certain items or conditions, has been shown in Experiment 3 . Instead of using irregular patterns, we increased the distractor variance by arranging different distractor types within a pattern. As was expected, an absent-advantage for homogeneous patterns occurred also when they were mixed with heterogeneous patterns. Moreover, as the results of Experiment 4 show, under these conditions it is even irrelevant whether the patterns are regular or not.

Taken together, our results demonstrate that an absentadvantage occurs for a specific type of pattern mainly when it is mixed with a certain other type. This suggests that decision processes play a major role for this phenomenon and have to be taken into account. If only the mixed conditions had been included in our experiments, we presumably would have concluded that homogeneity and regularity are necessary for an absent-advantage. By also considering constant conditions, we know that this is not the case. Rather, decisional processes are crucial. As our modeling shows, an absent-advantage can be produced simply by adjusting threshold values. No specific perceptual processes that occur in one condition but not in the other need be assumed. Nevertheless, certain perceptual conditions must be met for the specific criterion adjustments to take place. Although regularity and homogeneity are neither necessary nor sufficient for an absentadvantage, they are helpful. According to our account, these attributes reduce the variance and/or the mean of the activation produced by the distractors, relative to heterogeneous and irregular patterns.

Concerning our modeling, some details and assumptions were provisional, especially those with respect to the perceptual processes. However, the objective was not to provide a precise and complete model of visual search behavior, but to demonstrate that an absent-advantage can be produced simply by adjusting decision criteria. For attaining this goal, our model proved to be sufficient.

\section{REFERENCES}

Bamber, D. (1969). Reaction times and error rates for "same""different" judgments of multidimensional stimuli. Perception \& Psychophysics, 6, 169-174.

Chun, M. M., \& Wolfe, J. M. (1996). Just say no: How are visual searches terminated when there is no target present? Cognitive Psychology, 30, 39-78.

Donnelly, N., Humphreys, G. W., \& Riddoch, M. J. (1991). Parallel computation of primitive shape descriptions. Journal of Experimental Psychology: Human Perception \& Performance, 17, 561-570.

Duncan, J., \& Humphreys, G. W. (1989). Visual search and stimulus similarity. Psychological Review, 96, 433-458.

Duncan, J., \& Humphreys, G. W. (1992). Beyond the search surface: Visual search and attentional engagement. Journal of Experimental Psychology: Human Perception \& Performance, 18, 578-588.

Grossberg, S., Mingolla, E., \& Ross, W. D. (1994). A neural theory of attentive visual search: Interactions of boundary, surface, spatial, and object representations. Psychological Review, 101, 470-489.

HÜBNER, R. (in press). A formal version of the Guided Search model (GS2). Perception \& Psychophysics.

Humphreys, G. W., \& Müller, H. J. (1993). Search via recursive rejection (SERR): A connectionist model of visual search. Cognitive Psychology, 25, 43-110.

Humphreys, G. W., Quinlan, P. T., \& RidDOCH, M. J. (1989). Grouping processes in visual search: Effects with single- and combined-feature targets. Journal of Experimental Psychology: General, 118, 258-279.

KRUEGer, L. E. (1978). A theory of perceptual matching. Psychological Review, 85, 278-304.

Müller, H. J., Humphreys, G. W., \& Donnelly, N. (1994). Search via recursive rejection (SERR): Visual search for single and dual formconjunction targets. Journal of Experimental Psychology: Human Perception \& Performance, 20, 235-258.

PAshler, H. (1987). Detecting conjunctions of color and form: Reassessing the serial search hypothesis. Perception \& Psychophysics, 41, 191-201.

Prinzmetal, W., \& Banks, W. P. (1977). Good continuation affects visual detection. Perception \& Psychophysics, 21, 389-395.

Strayer, D., \& Kramer, A. F. (1994). Strategies and automaticity: II. Dynamic aspects of strategy adjustment. Journal of Experimental Psychology: Learning, Memory, \& Cognition, 20, 342-365.

Treisman, A. (1988). Features and objects: The 14th Bartlett Memorial Lecture. Quarterly Journal of Experimental Psychology, 40A, 201-237.

Treisman, A., \& Gelade, G. (1980). A feature-integration theory of attention. Cognitive Psychology, 12, 97-136.

Treisman, A., \& Gormican, S. (1988). Feature analysis in early vision: Evidence from search asymmetries. Psychological Review, 95, 15-48.

Treisman, A., \& Souther, J. (1985). Search asymmetry: A diagnostic for preattentive processing of separable features. Journal of Experimental Psychology: General, 3, 285-310.

Treisman, M., \& Williams, T. C. (1984). A theory of criterion setting with an application to sequential dependencies. Psychological Review, 91, 68-111.

Wang, Q., CAVAnagh, P., \& Green, M. (1994). Familiarity and pop-out in visual search. Perception \& Psychophysics, 56, 495-500.

Wolfe, J. M. (1994). Guided Search 2.0: A revised model of visual search. Psychonomic Bulletin \& Review, 1, 202-238.

Wolfe, J. M. (1998). What can 1 million trials tell us about visual search? Psychological Science, 9, 33-39.

\section{APPENDIX}

In GS2 it is assumed that target and distractor activations on the activation map can be represented by random variables $X_{s}$ and $X_{n}$, respectively. The corresponding densities will be denoted by $f_{s}(x)$ and $f_{n}(x)$, and the distribution functions by $F_{s}(x)$ and $F_{n}(x)$. The crucial assumption, however, is that the items are checked serially in descending order of their activation strength until the target is found or an activation threshold is reached. Thus, when the target is above the activation threshold, the search time $T$ depends only on the number of distractors whose activation exceeds that of the target. Let $R$ denote a discrete random variable representing this number, and $t h_{a}$ the activation threshold. For a set size of $m$ items, the probability mass function of $R$ (see Hübner, in press) is given by:

$$
P_{m}\{R=r\}=\frac{1}{1-F_{s}\left(t h_{a}\right)}\left(\begin{array}{c}
m-1 \\
r
\end{array}\right) \int_{t h_{a}}^{\infty}\left[1-F_{n}(x)\right]^{r}\left[F_{n}(x)\right]^{m-1-r} f_{s}(x) d x, r=0,1, \ldots, m-1 .
$$


On target-absent trials, the search time is determined by the number of distractors whose activation is above the activation threshold. Let $N$ denote a random variable representing this number. It has a binomial distribution with parameters $(m, q)$ :

$$
P_{m}\{N=k\}=\left(\begin{array}{c}
m \\
k
\end{array}\right) q^{k}(1-q)^{m-k}, \quad k=0,1, \ldots, m,
$$

where $q$ is the probability that a single distractor activation is above threshold:

$$
q=1-F_{n}\left(t h_{a}\right) .
$$

Given these probability mass functions, one can easily compute the expected search times for present-responses and absent-responses according to the standard GS2 model (see Hübner, in press). However, since this model cannot explain an absent-advantage, we introduce two further thresholds: A no-threshold $t h_{o}$ and a yes-threshold $t h_{y}$, where $t h_{y} \geq$ $t h_{a} \geq t h_{o}$. It is assumed that when no activation passes the no-threshold, an absent-response is triggered immediately. On the other hand, when at least one activation is above the yesthreshold, a present-response is triggered without delay. Thus, guided search takes place only when some activations are above the no-threshold but no activation is larger than the yes-threshold.

\section{Present-Responses}

Let us first consider the case in which a target is present. When the target activation is below the activation threshold and when all distractor activations are below the yesthreshold, we have a miss. Thus, a miss occurs with probability

Accordingly, the probability of a hit is

$$
P_{m}(\text { Miss })=F_{s}\left(t h_{a}\right)\left[F_{n}\left(t h_{y}\right)\right]^{m-1} \text {. }
$$

$$
P_{m}(\text { Hit })=1-P_{m}(\text { Miss }) \text {. }
$$

The responses corresponding to hits can be separated into fast and slow ones. A fast response is triggered when at least one of the $m$ activations is above the yes-threshold. Let FH (fast hit) denote this event. It occurs with probability

$$
P_{m}(\mathrm{FH})=1-F_{s}\left(t h_{y}\right)\left[F_{n}\left(t h_{y}\right)\right]^{m-1} .
$$

For the case in which all activations are below the yes-threshold and the target activation is above the activation threshold, we have guided search ( $\mathrm{SH}$, slow hit), which occurs with probability

$$
P_{m}(\mathrm{SH})=\left[F_{s}\left(t h_{y}\right)-F_{s}\left(t h_{a}\right)\right]\left[F_{n}\left(t h_{y}\right)\right]^{m-1} .
$$

In this case, the search time depends on the number, $R$, of distractor activations above the target activation. For deriving the probability mass function of $R$, we have to consider only the case in which the target activation is between the activation threshold and the yes-threshold and all distractors are below the yes-threshold. Thus, it is given by

$$
P_{m}\{R=r\}=\frac{\left(\begin{array}{c}
m-1 \\
r
\end{array}\right)_{t h_{a}}^{t h_{y}}\left[F_{n}\left(t h_{y}\right)-F_{n}(x)\right]^{r}\left[F_{n}(x)\right]^{m-1-r} f_{s}(x) d x}{\left[F_{s}\left(t h_{y}\right)-F_{s}\left(t h_{a}\right)\right] \times\left[F_{n}\left(t h_{y}\right)\right]^{m-1}}, r=0,1, \ldots, m-1 .
$$

To compute the expected search time for the correct present-responses, we have to take both response types into account. The expected response time for the fast hits is

$$
E_{\mathrm{FH}}\left[T_{m}\right]=\text { offset, }
$$

where offset represents a fixed amount of time used for preliminary perceptual processes and for performing the response. For the guided search trials, the expected search time is, with $P_{m}\{R=r\}=p_{m}(r)$,

$$
E_{\mathrm{SH}}\left[T_{m}\right]=\operatorname{off} s e t+t \times \sum_{i=0}^{m-1} i p_{m}(i),
$$

where $t$ is the time per processed item.

Finally, by combining the expected values for both response types, we obtain the formula for the expected response time for the correct present-decisions. It is

$$
E\left[T_{m}\right]=E_{\mathrm{FH}}\left[T_{m}\right] \frac{P_{m}(\mathrm{FH})}{P_{m}(\mathrm{Hit})}+E_{\mathrm{SH}}\left[T_{m}\right] \frac{P_{m}(\mathrm{SH})}{P_{m}(\mathrm{Hit})} .
$$




\section{Absent-Responses}

Now, we consider the responses on trials on which no target is present. In this case, an incorrect target-presentresponse is triggered when an activation is above the yes-threshold. That is, the false alarm (FA) rate for a set size of $m$ is given by

$$
P_{m}(\mathrm{FA})=1-\left[F_{n}\left(t h_{y}\right)\right]^{m} .
$$

The probability of a correct absent-response (CR, correct rejection) is, accordingly,

$$
P_{m}(\mathrm{CR})=1-P_{m}(\mathrm{FA}) \text {. }
$$

As for hits, the correct absent-responses also can be separated into fast and slow responses. A fast absent-response (FR, fast rejection) occurs when no activation is above the no-threshold. Its probability is

$$
P_{m}(\mathrm{FR})=\left[F_{n}\left(t h_{o}\right)\right]^{m} .
$$

The expected response time $E_{\mathrm{FR}}\left[T_{m}\right]$ for this rejection type is simply the offset, introduced above.

When some activations are above the no-threshold but no activation is above the yesthreshold, we have guided search (SR, slow rejection). This occurs with probability

$$
P_{m}(\mathrm{SR})=1-P_{m}(\mathrm{FA})-P_{m}(\mathrm{FR}) .
$$

The response time for this case depends on the number, $N$, of activations above the activation threshold. This number has a binomial distribution, $P_{m}\{N=k\}$, with parameters $(m, q)$, where $q$ is, in this case,

$$
q=F_{n}\left(t h_{y}\right)-F_{n}\left(t h_{a}\right) .
$$

The expected response time for this rejection type is, with $P_{m}\{N=k\}=p_{m}(k)$,

$$
E_{\mathrm{SR}}\left[T_{m}\right]=\text { offset }+t \times \sum_{k=0}^{m} k p_{m}(k), k=0,1, \ldots, m .
$$

To compute the expected response time for correct absent-responses, we have to combine the expected times for the two rejection types:

$$
E\left[T_{m}\right]=E_{\mathrm{FR}}\left[T_{m}\right] \frac{P_{m}(\mathrm{FR})}{P_{m}(\mathrm{CR})}+E_{\mathrm{SR}}\left[T_{m}\right] \frac{P_{m}(\mathrm{SR})}{P_{m}(\mathrm{CR})} .
$$

